Provided for non-commercial research and education use. Not for reproduction, distribution or commercial use.

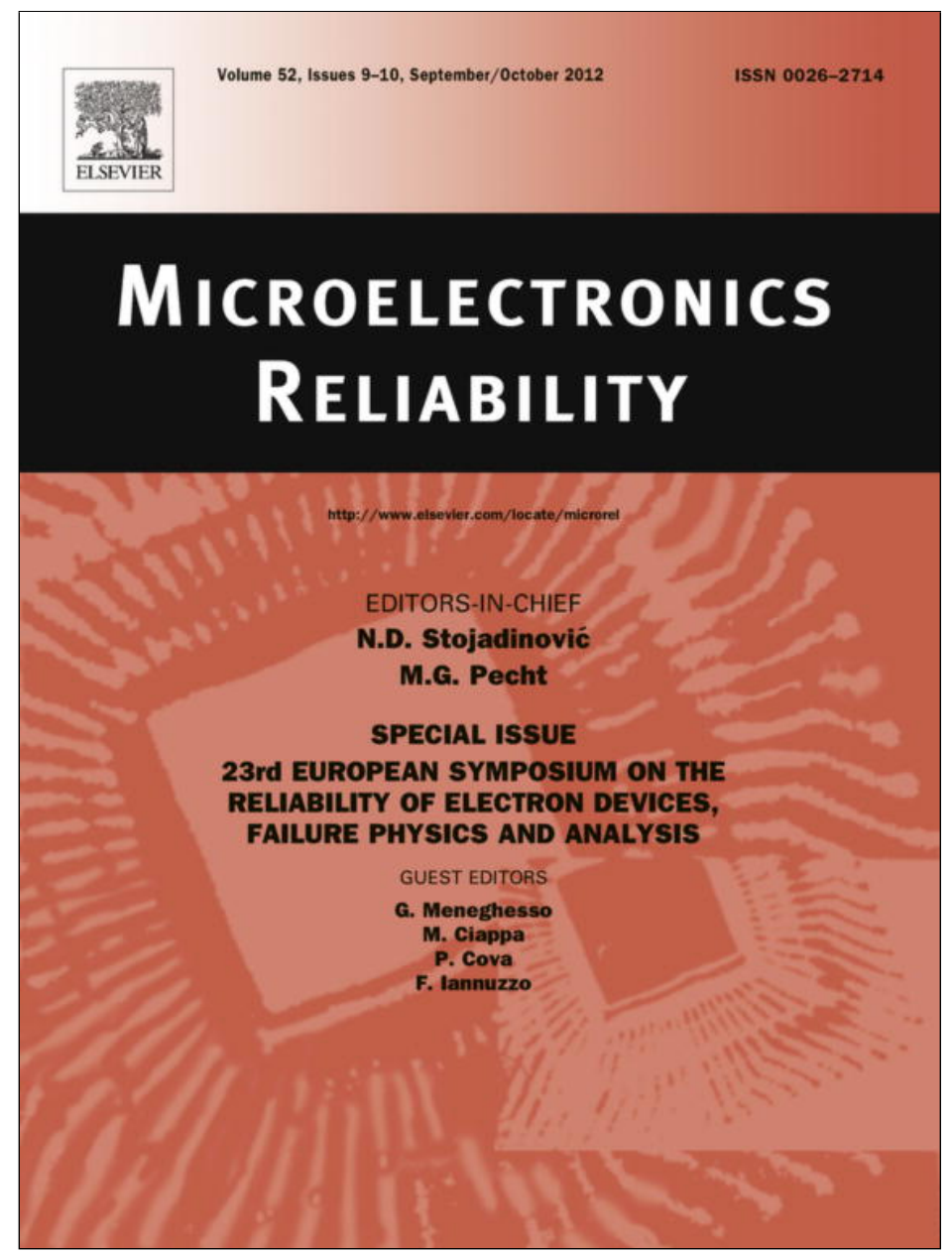

This article appeared in a journal published by Elsevier. The attached copy is furnished to the author for internal non-commercial research and education use, including for instruction at the authors institution and sharing with colleagues.

Other uses, including reproduction and distribution, or selling or licensing copies, or posting to personal, institutional or third party websites are prohibited.

In most cases authors are permitted to post their version of the article (e.g. in Word or Tex form) to their personal website or institutional repository. Authors requiring further information regarding Elsevier's archiving and manuscript policies are encouraged to visit:

http://www.elsevier.com/copyright 


\title{
Temperature effects on the bulk discharge current of dielectric films of MEMS capacitive switches
}

\author{
M. Koutsoureli *, L. Michalas, G. Papaioannou \\ Solid State Physics Section, Physics Department, National and Kapodistrian University of Athens, Panepistimioupolis, Zografos, Athens 15784, Greece
}

\section{A R T I C L E I N F O}

\section{Article history:}

Received 30 May 2012

Accepted 9 June 2012

Available online 29 June 2012

\begin{abstract}
A B S T R A C T
Kelvin probe method has been directly applied to capacitive MEMS switches in order to investigate temperature activated mechanisms in PECVD Silicon Nitride $\left(\mathrm{SiN}_{x}\right)$ films. The bulk discharge current of MEMS capacitive switches has been determined for different charging and discharging temperatures, in the range of 300-400 K. The increase of discharging temperature leads to an increase of the magnitude of the bulk discharge current and the relaxation time of the discharging process is found to be thermally activated. Finally, it is shown that the increase of charging temperature assists trapping at centers characterized by time constants even longer than the time window of observation, i.e. $10^{4} \mathrm{~s}$.
\end{abstract}

(c) 2012 Elsevier Ltd. All rights reserved.

\section{Introduction}

Capacitive RF MEMS switches are very promising components for radio frequency applications but their commercialization is still hindered by reliability problems, the most important being the effect of dielectric charging [1]. The dielectric films used in MEMS switches are highly disordered materials, which deviate from stoichiometry and contain a large density of dipoles and defects [2-4]. The presence of high electric fields during actuation results to charge injection in the dielectric films, fact that causes erratic device behavior and limits the device lifetime [5]. In MEMS capacitive switches it has been demonstrated that the charge trapping and emission as well as dipole orientation are thermally activated [6]. Thus the charging and discharging processes in the bulk of the dielectric film is expected to be strongly affected by temperature. Based on this phenomenon an active heat-based restoring mechanism has been recently proposed in order to increase the switches reliability [7].

So far, the discharging process has been investigated in MetalInsulator-Metal (MIM) capacitors with the aid of thermally stimulated depolarization currents (TSDC) method [8-10], by recording the charging and discharging current transients (CCT and DCT) [11] and recently with the aid of Kelvin probe force microscopy (KPFM) method [12,13]. In capacitive MEMS switches the discharging process differs from the one in MIM capacitors, since in MEMS the injected charges are collected only through the bottom electrode when the suspended electrode is in the pull-up state. In order to investigate the discharge in MEMS switches, the KPFM method [12-14] and the shift of bias at minimum up-state capacitance

\footnotetext{
* Corresponding author. Tel.: +30 210727 6722; fax: +30 2107276711.

E-mail address: mkoutsoureli@phys.uoa.gr (M. Koutsoureli).
}

[10] have been used. In addition, the discharge current transient in MEMS switches has been calculated for a time in excess of $10^{4} \mathrm{~s}$ [15]. Up to now, the bulk discharge current transient has been found to depend on the stressing field's polarity and intensity as well as on stressing time [16,17]. Regarding the charge collection the dominant mechanism has been found to be the variable range hopping [17], which is strongly dependent on temperature.

The aim of the present work is to present a systematic study of the thermally activated bulk discharge mechanisms in MEMS capacitive switches. Taking into account that the spatial and energy distribution of trapped charges is determined by the charging temperature, the study has been implemented by stressing the devices at room temperature, like in conventional MEMS operation, while the bulk discharge current was monitored at different temperatures, ranging from $300 \mathrm{~K}$ to $400 \mathrm{~K}$. The impact of charging at different temperatures has been also investigated after stressing the devices at different temperatures, from $300 \mathrm{~K}$ to $400 \mathrm{~K}$, and monitoring the bulk discharge currents at the same temperature. In all cases, the experimental results reveal the presence of two discharge mechanisms, one that occurs within the time window of experiment and a second one with a much longer relaxation time.

\section{Theoretical background}

\subsection{Charging process}

The basic mechanisms involved in the charging process are the Trap-Assisted-Tunnelling (TAT) and the transient component of Poole-Frenkel (PF) effect that is responsible for the charge redistribution [18]. The hopping conduction, although present, plays a rather minor role in the presence of high electric fields [19]. The 
simultaneous action of the two mechanisms leads to a spatial charge distribution that was presented for first time in [20] and practically confirmed by a similar one, obtained by a different numerical approach and presented in [21].

The contribution of TAT is calculated using the WentzelKramers-Brillouin (WKB) approximation [22] for the tunneling probability $P$ :

$P=\exp \left(-2 \int|k(x) d x|\right)$

and $k(x)$ is given by:

$P=\left[\frac{2 q m}{h^{2}}\left(\Phi-F x-E_{e}\right)\right]^{1 / 2}$

where $m$ is the electron effective mass in the dielectric film, $q$ the elementary charge, $\hbar$ the reduced Plank constant, $\Phi$ the barrier height of the tunneling, $F$ the electric field intensity and $E_{e}$ is the total electron energy in the metal (taken as $0.2 \mathrm{eV}$ [23]). Further adopting the formulation proposed by Ramprasad [18] the time dependent current, assuming that TAT is the only operating mechanism, is given by:

$j_{\text {TAT }}(t)=q \int_{x=0}^{\infty} \int_{E=-\infty}^{-q F x} N^{f f}(x, E+q F x) \cdot\left(f_{\infty}-f_{0}\right) \cdot \frac{e^{-t / \tau}}{\tau} \cdot \frac{x}{L} d E \cdot d x$

where the current flows in the $x$ direction, $L$ is the sample thickness, $N_{f f}$ the field free trap distribution (in our case uniform or exponential), $E$ the energy, $F$ the electric field intensity, $f_{\infty}$ and $f_{0}$ are the Fermi functions:

$$
\begin{aligned}
& f_{\infty}(E)=\left\{1+\exp \left[\left(E-E_{F}\right) / k T\right]\right\}^{-1}, \\
& f_{0}(E)=\left\{1+\exp \left[\left(E+q F x-E_{F}\right) / k T\right]\right\}^{-1} \text { and } \tau=\tau_{0} e^{2 K x}
\end{aligned}
$$

with $k, T$ and $E_{F}$ being the Boltzmann's constant, the temperature and the Fermi energy of the metal electrode respectively. The injected charge is assumed to be redistributed by the transient component of Poole-Frenkel conduction, which is derived assuming that a certain fraction of the trapped electrons are lost to PF emission, thus decreasing the density of injected/trapped charges through the TAT mechanism. Since there is a distribution of trap states, the transient component of Poole-Frenkel current density was defined [18] as:

$j_{\text {TPF }}(t)=q \mu F \int_{x=0}^{\infty} \int_{E=-\infty}^{-q F x} \exp \left\{-\frac{q}{k T}\left(E-\sqrt{\frac{q F}{\pi \varepsilon_{o p t}}}\right)\right\} \cdot n(x, E, t) \cdot d E \cdot d x$

where $\varepsilon_{o p t}$ is the high frequency dielectric constant, $n$ the density of trapped electrons at a depth $E$ from the conduction band and the rest parameters are the same as described earlier. Here it must be pointed out that at any given time the PF process alters the trapped distribution of electrons, which in turn alters the TAT process at subsequent times. This obviously means that the simulation of dielectric charging must take into account these mechanisms simultaneously, as proposed in [18].

The simultaneous action of TAT and Poole-Frenkel current transients on the distribution of injected and trapped charges has been calculated for $\operatorname{SiN}_{x}$ assuming band tails proportional to $\exp \left(-E / E_{0}\right)$ with $E_{0} \approx 0.1 \mathrm{eV}$ [20]. The later was assumed to behave in a similar manner like amorphous silicon [24,25]. A realistic value for the band gap of the insulating film was assumed to be $3.4 \mathrm{eV}$ [26], approximating the one of HF PECVD $\mathrm{SiN}_{x}$ and the Fermi level was placed to mid gap [27]. The calculations lead to a distribution of charge that depends on injection time as shown in Fig. 1. Here it must be strongly emphasized that the spatial distribution of trapped charge depends on both the applied electric field and temperature.

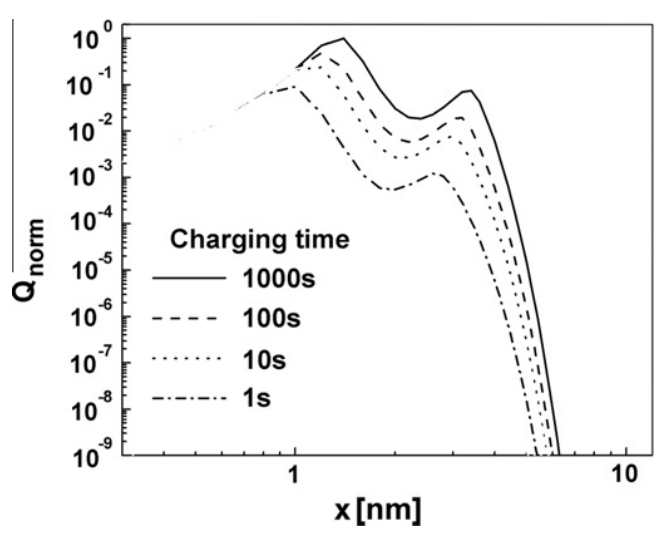

Fig. 1. Distribution of normalized charge density, with respect to $1000 \mathrm{~s}$, for different charging times [20].

\subsection{Discharging process}

The discharging process in the dielectric film of a MEMS capacitive switch takes place under an intrinsic electric field, which is lower than the injecting one and decreases continuously with time [17]. The dominant mechanism during this process has been found to be variable range hopping $[17,19]$, which is strongly affected by temperature.

According to a traditional approach to the analysis of charge carrier kinetics in disordered hopping systems, the carrier jump rate $v$ from a starting site of energy $E_{s}$ to a target site of energy $E_{t}$ over the distance $r$ is $[28,29]$ :

$v=v_{0} \cdot \exp (-u)$

where $u$ is a hopping parameter defined as:

$u\left(E_{s}, E_{t}, r\right)=2 \gamma r+ \begin{cases}0, & E_{t}<E_{s}+e F r z \\ \frac{E_{t}-E_{s}-e F r z}{k_{B} T}, & E_{t}>E_{s}+e F r z\end{cases}$

here $F$ is the intensity of the electric field, $T$ the temperature, $v_{0}$ the attempt to jump frequency, $\gamma$ the inverse localization radius, $e$ the elementary charge, $k_{B}$ is the Boltzmann's constant and $z=\cos \theta$ with $\theta$ being the angle between the field and the jump direction. The hopping parameter clearly shows that the jump rate is determined by temperature and by the presence and magnitude of electric field intensity, but only if the latter is strong enough in order to significantly change the energy difference between starting and target sites. Moreover the distribution of trapping states in the band gap plays a key issue role on the application of Eq. (6).

The temperature dependence of dc conductivity in a disordered system has been predicted by Mott [30] and it has been found to be consistent with the law:

$\sigma \propto \exp \left[-\left(\frac{\lambda \cdot \alpha^{3}}{N\left(E_{F}\right) \cdot k_{B} \cdot T}\right)^{1 / 4}\right]$

where $N\left(E_{F}\right)$ is the density of states at the Fermi level, $\alpha^{-1}$ the distance for exponential decay of the wave functions and $\lambda$ is the dimensionless constant, which has been estimated to be approximately 16 [31].

\section{Experimental procedure}

The switches used in the present work (Fig. 2a) are unpackaged bridge-type capacitive switches, fabricated with a standard photolithographic process on high resistivity silicon wafers on top of which a $3 \mu \mathrm{m} \mathrm{SiO}{ }_{2}$ film was deposited. The $\mathrm{SiN}_{x}$ dielectric film was grown with PECVD method at $300{ }^{\circ} \mathrm{C}$ and the thickness of the film is $250 \mathrm{~nm}$. The membrane is an evaporated titanium-gold 
(a)

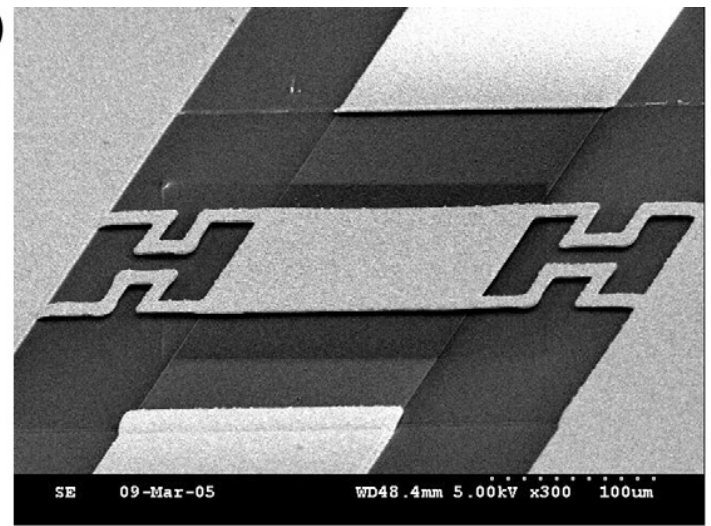

(b)

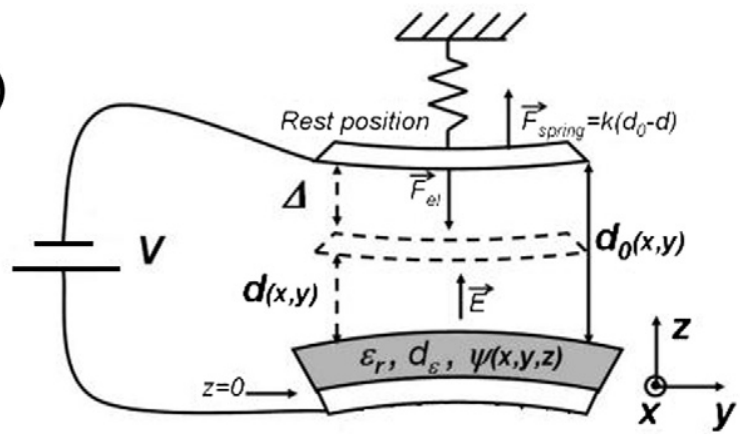

Fig. 2. (a) Top view of the utilized switches and (b) model of a capacitive switch with non-uniform trapped charge and air gap distributions [33] (๔ [2007] IEEE).

seed layer electroplated to a thickness of $2.0 \mu \mathrm{m}$ and in the unactuated position it is suspended about $2.2 \mu \mathrm{m}$ above the dielectric. The active area of the switches is $2.5 \times 10^{-5} \mathrm{t} \mathrm{cm}^{2}$.

Taking into account that the spatial and energy charge distribution of trapped charges as well as hopping conductivity depend on temperature we performed two experiments. First the devices were stressed at $300 \mathrm{~K}$, simulating the conventional operation of a MEMS switch, and the discharge was monitored at different temperatures, in the range of $300-400 \mathrm{~K}$. In the second experiment the charging was performed at different temperatures and the discharge was monitored at a selected temperature. The later was done in order to study the effect of temperature on charging process. In all cases the stressing time was $5 \mathrm{~min}$ at $30 \mathrm{~V}$ bias, while the pull-in voltage of the switches at room temperature was $V_{p i}=20 \mathrm{~V}$. After stress the devices were assessed by obtaining the capacitance-voltage $(C-V)$ characteristics in the up state for $10^{4} \mathrm{~s}$ in order to monitor the shift of the bias for minimum capacitance towards the pre-stress level.

The up-state $C-V$ characteristics were monitored with a Boonton 72B capacitance meter while sweeping the voltage in $50 \mathrm{mV}$ steps and the acquired bias was applied by a 6487 Keithley voltage source - picoampere meter. The bias for up state capacitance minimum was determined by fitting a parabola to the experimental data, assuming a very small capacitance variance. Finally, all measurements have been performed in a BioRad Cryostat under vacuum with prior $2 \mathrm{~h}$ annealing at $140{ }^{\circ} \mathrm{C}$, in order to avoid any interference from humidity [32].

The bulk discharge current transient in MEMS capacitive switches is determined using the Kelvin Probe Force (KPF) method, proposed in $[15,16]$. This method adopts the device model proposed in [33] of a real MEMS switch with non-uniform charge and air gap distributions (Fig. 2b). According to KPF method, the up-state capacitance attains its minimum at the bias $\left(V_{\min }\right)$ for which the electrostatic force is minimum and for small bridge up-state deformation, where the capacitance variance can be considered low, the bias $V_{\min }$ is given by:

$V_{\min }(t)=\frac{d_{\varepsilon}}{\varepsilon_{r} \cdot \varepsilon_{0}} \cdot \mu_{\psi}$

The average value of discharge current density due to charge collection by the bottom electrode is then found $[15,16]$ to be:

$J_{\text {disch }}(t)=-\frac{d \mu_{\psi}(t)}{d t}=-\frac{\varepsilon_{r} \varepsilon_{0}}{d_{\varepsilon}} \cdot \frac{d V_{\min }(t)}{d t}$

Here $\mu_{\psi}$ represents the mean value of the equivalent surface charge distribution, $d_{\varepsilon}$ is the (uniform) thickness of the dielectric film with dielectric constant $\varepsilon_{r}$ and $\varepsilon_{0}$ is the vacuum permittivity. The transported charge density $\sigma_{\text {disch }}$ during discharge process can be calculated by integrating the discharge current density within the time window of observation:

$\sigma_{\text {disch }}=\int_{t_{0}}^{t} J_{\text {disch }} \cdot d t$

\section{Results and discussion}

The dielectric film in the utilized MEMS switches is amorphous silicon nitride, so the charge decay, arising from dipole and space charge polarization mechanisms, is expected to obey a stretched exponential law of the form:

$V_{m}(t)=\Delta V_{0} \cdot \exp \left[-\left(\frac{t}{\tau}\right)^{\beta}\right]+V_{\text {offset }}$

where $\Delta V_{0}=\frac{d_{\varepsilon}}{\varepsilon_{r} \varepsilon_{0}} \cdot \Delta \mu_{\psi, 0}, \Delta \mu_{\psi, 0}$ is a fitting parameter representing the mean value of the transported charge distribution (at $t=0 \mathrm{~s}$ ) and $V_{\text {offset }}$ is the potential offset during the time window of the present experiment, arising from charges which are collected under very long time constants (greater than the time window of observation, i.e. $10^{4} \mathrm{~s}$ ).

After stressing the devices at $300 \mathrm{~K}$ we monitored the transients of $V_{\min }$ during discharge at different temperatures.

In all cases two basic discharging mechanisms appear. One that takes place within the time window of our experiment and gives rise to the bulk discharge current we have calculated and another one with very long relaxation time (longer than the time window of observation) that give rise to an offset $\left(V_{\text {offset }}\right)$ on the transients of $V_{\min }$. In Fig. 3a the transient of $V_{\min }$ due to the discharging mechanism that takes place within the time window of observation is presented. In this figure $\Delta V_{\min }$ refers to the discharging mechanism described above and so $\Delta V_{\min }=V_{\min }-V_{\text {offset }}$.

Moreover, the fitting of the experimental data with Eq. (11) revealed (Fig. 3b) a discharging mechanism that appears within the time window of observation with a thermally activated relaxation time:

$\tau(T)=\tau_{0} \cdot \exp \left(\frac{E_{A}}{k_{B} T}\right)$

where $k_{B}$ is the Boltzmann's constant, $T$ is the temperature, $\tau_{0}=7.5 \times 10^{-2} \mathrm{~s}$ is the inverse vibrational frequency and the activation energy is found to be $E_{A}=0.26 \mathrm{eV}$.

The bulk discharge current that was calculated from Eq. (9) for different discharging temperatures is presented in Fig. 4 and its magnitude is found to increase with temperature. This is attributed to the fact that the discharging process in MEMS capacitive switches is dominated by variable range hopping mechanism $[17,19]$. It is then expected that when temperature increases more charges will be transported, leading to an increase of bulk 

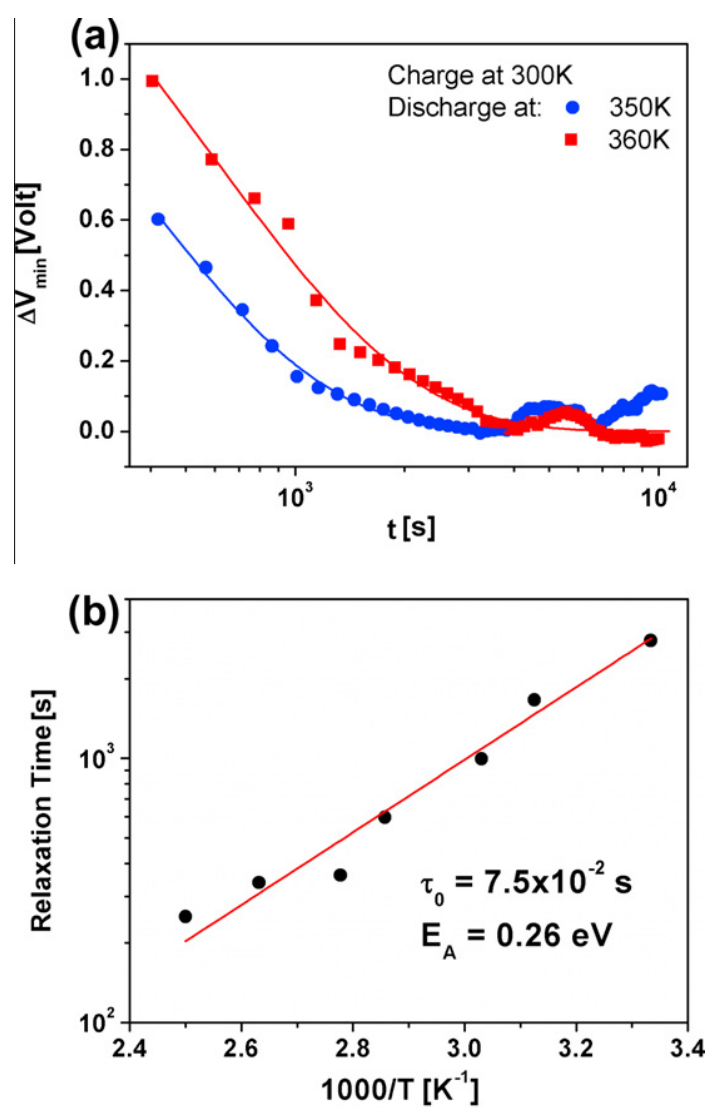

Fig. 3. (a) Transients of $\Delta V_{\text {min }}=V_{\text {min }}-V_{\text {offset }}$, for the discharging mechanism that takes place within the time window of observation, at two different discharging temperatures and (b) Arrhenius plot of relaxation times of $V_{\text {min }}$, obtained from the fit of experimental results with Eq. (11)

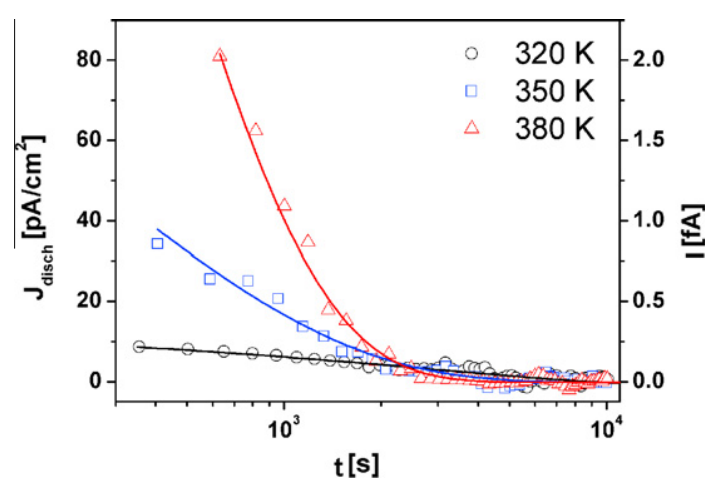

Fig. 4. Determined bulk discharge current at different discharge temperatures. The devices were stressed at $300 \mathrm{~K}$ in all cases.

discharge current and this process is expected to take place in a shorter time.

The transported charge density within the time window of observation was also calculated for each discharging temperature using Eq. (10) and it is presented in Fig. 5. This charge density has been found to be consistent with the law:

$\sigma_{\text {disch }}(T) \propto \exp \left(-\frac{E_{A}}{k_{B} T}\right)$

where the activation energy is $E_{A}=0.41 \mathrm{eV}$. This activation energy practically coincides with the one obtained from TSDC method in

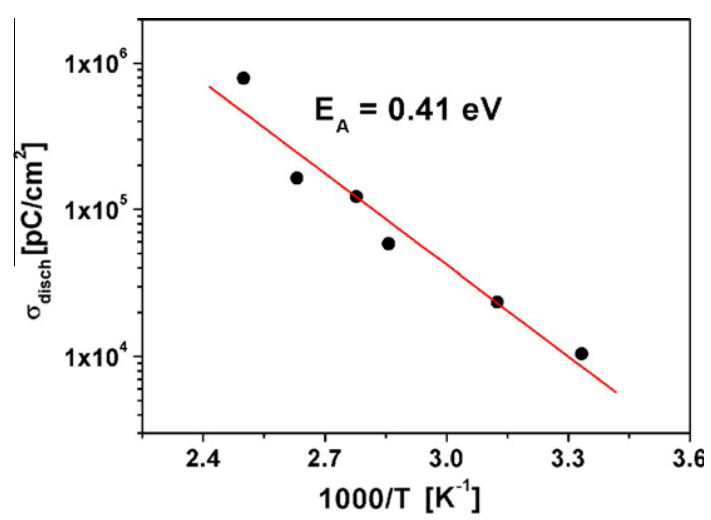

Fig. 5. Arrhenius plot of the calculated transported charge density, after stressing the devices at $300 \mathrm{~K}$.

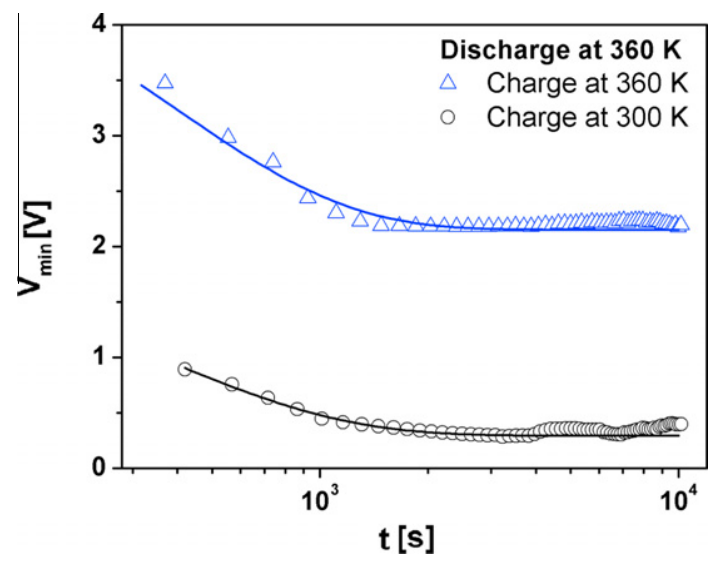

Fig. 6. Transients of $V_{\min }$ when the discharge process takes place at $360 \mathrm{~K}$ and charging occurs at $300 \mathrm{~K}$ and $360 \mathrm{~K}$.

MIM capacitors [9], where above room temperature the TSDC current can be described by:

$I_{T S D C}(T) \propto \tau_{0 \infty}^{-1} \exp \left(-E_{A} / k_{B} T\right)$

where $E_{A}$ is the activation energy and $\tau_{0 \infty}$ the time constant for assuming an infinite number of relaxing Debye sub-processes [34]. Under such circumstances the TSDC current has an envelope that exhibits Arrhenius-like behavior, which has been attributed to the emission of trapped charges from a continuous distribution of trap states in the dielectric film. So, the calculated activation energy (Fig. 5) may refer to the envelope of such a distribution of Debye relaxing sub-processes. It is important to mention that the TSDC method differs from the Kelvin probe method in MEMS, used in this work, and it is not easy to directly correlate the two assessment methods.

Finally, the discharge process has been investigated for different charging temperatures. This has been done because the charging is a thermally activated process [10] and the increase of temperature enhances charging effects. The experimental results revealed that the increase of charging temperature assists trapping at centers characterized by time constants even longer than the time window of observation. This is easily observed from the increase of $V_{\text {offset }}$ on transients of $V_{\min }$ (Fig. 6). These traps are expected to contribute significantly on the lifetime of MEMS switches. The existence of such "fixed" charges has been also reported in MISS devices with an a-SiN $\mathrm{N}_{x}$ :H gate quality insulator deposited with PECVD method at $260{ }^{\circ} \mathrm{C}$ [35], where the shift of $C-V$ characteristics after positive stress revealed a thermally activated mechanism with $\tau=3.6 \times$ 
$10^{10} \mathrm{~s}$. This mechanism has been attributed not to the creation of dangling bond defect states in a-Si:H but to trap states in the a-SiN $\mathrm{N}_{x}: \mathrm{H}$ insulator [35].

The activation energies of the discharging process obtained in this work is in excellent agreement with our previously reported results $[9,19]$ as far as it concerns thermally activated charging mechanisms in $\operatorname{SiN}_{x}$. The fact that charging and discharging processes in MEMS switches are thermally activated means that the parameters affecting the device lifetime are thermally activated too. This has a significant impact on the reliability of MEMS since it allows us to detect the contributions responsible for the device ageing from their "signature", which is the Arrhenius plot.

\section{Conclusions}

The present paper introduces an appropriate procedure for the use of temperature in order to study dielectric charging. Taking into account that mechanisms that dominate charging and discharging processes are affected significantly by temperature, it is important to use a method that separates the contributions from each process. In the proposed methodology, charging takes place at a given temperature in order to ensure that the amount of injected charge and its spatial distribution depends only on the stress bias and time. Then the following discharge is performed at different temperatures in order to investigate the thermally activated mechanisms that affect the charge collection process. The determined activation energy for relaxation time and transported charge density results from the percolative hopping conduction in the disordered dielectric film of the utilized switches.

Thus the application of Kelvin Probe method on $\mathrm{SiN}_{x}$ films of MEMS capacitive switches reveals the presence of one thermally activated mechanism that occurs within the time window of experiment, i.e. $10^{4} \mathrm{~s}$, and a second one with a much longer relaxation time. The increase of discharging temperature results to an increase of the magnitude of the bulk discharge current due to the increase of hopping conductivity.

Finally, in order to investigate the effect of charging temperature on the dielectric charging, the devices are stressed at different temperatures while the following discharging process takes place at a given temperature. The experimental results clearly show that the increase of charging temperature assists charge trapping at states characterized by time constants much longer than the time window of the experiment, which are expected to play a significant role to the device lifetime.

\section{Acknowledgments}

The present work has been supported by ENIAC-2010 NANOCOM project and co-financed by the European Union (European Social Fund - ESF) and Greek national funds through the Operational Program "Education and Lifelong Learning" of the National Strategic Reference Framework (NSRF) - Research Funding Program: "Heracleitus II Investing in knowledge society through the European Social Fund".

\section{References}

[1] Wibbeler J, Pfeifer G, Hietschold M. Sens Actuators A 1998;71:74-80.

[2] Krick DT, Lenahan PM, Kanicki J. J Appl Phys 1998;64(7):3558-63.

[3] Gritsenko VA, Gritsenko DV, Novikov YN, Kwok RWM, Bello I. J Exp Theor Phys 2004;98:760-9.

[4] Papaioannou G. The impact of dielectric material and temperature on dielectric charging in RF MEMS capacitive switches. In: Gusev E, Gurfankel E, Dideikin A editors. NATO science for peace and security series - B: Physics and biophysics. Springer; 2009. p. 141-53

[5] Goldsmith C, Ehmke J, Malczewski A, Pillans B, Eshelman S, Yao Z, et al. IEEE MTT-S Digest 2011:227-30.

[6] Papaioannou G, Exarchos M, Theonas V, Wang G, Papapolymerou J. IEEE Trans Microwave Theory Tech 2005;53:3467-73.

[7] Iannacci J, Faes A, Repchankova A, Tazzoli A, Meneghesso G. Microelectron Reliab 2011;51:1869-73.

[8] Daigler R, Papandreou E, Koutsoureli M, Papaioannou G, Papapolymerou J. Microelectron Eng 2009;86:404-7.

[9] Koutsoureli M, Papandreou E, Michalas L, Papaioannou G. Microelectron Eng 2012;90:145-8.

[10] Papaioannou G, Papapolymerou J, Pons P, Plana R. Appl Phys Lett 2007;90: 233507.

[11] Lamhamdi M, Pons P, Zaghloul U, Boudou L, Coccetti F, Guastavino J, et al. Microelectron Reliab 2008;48:1248-52.

[12] Zaghloul U, Koutsoureli M, Wang H, Coccetti F, Papaioannou G, Pons P, et al. Microelectron Reliab 2010;50:1615-20.

[13] Zaghloul U, Papaioannou GJ, Wang H, Bhushan B, Coccetti F, Pons P, et al. Nanotechnology 2011;22(20):205708.

[14] Herfst RW, Steeneken PG, Schmitz J, Mank AJG, Van Gils M. In: 46th Annua international reliability physics symposium, Phoenix, Arizona, USA; 2008. p. 492-5.

[15] Koutsoureli M, Papaioannou G. Appl Phys Lett 2011;99:103503.

[16] Koutsoureli MS, Papaioannou GJ. Microelectron Reliab 2011;51:1874-7.

[17] Koutsoureli M, Michalas L, Papaioannou G. In: IEEE international reliability physics symposium (IRPS). Anaheim, CA, USA; April 15-19 2012.

[18] Ramprasad R. Phys Status Solidi (b) 2003;239(1):59-70.

[19] Koutsoureli M, Michalas L, Papaioannou G. In: IEEE international reliability physics symposium (IRPS). Monterey, CA, USA; April 10-14 2011.

[20] Papaioannou G, Coccetti F, Plana R. Topical meeting on silicon monolithic integrated circuits in RF systems (SiRF). N. Orleans, LA, USA, January 11-13 2010.

[21] Palit S, Alam M. J Appl Phys 2012;111:054112.

[22] Oh SJ, Yeow YT. Solid-State Electron 1989;32:507-11.

[23] Svensson C, Lundstrom I. J Appl Phys 1973;44:4657-63.

[24] Brinza M, Emelianova EV, Adriaenssens GJ. Phys Rev B 2005;71:115209.

[25] Altermatt PP, Heiser G. J Appl Phys 2002;92:2561-74.

[26] Robertson J. Philos Mag B 1991;63:47-77.

[27] Papandreou E, Lamhamdi M, Skoulikidou CM, Pons P, Papaioannou GJ, Plana R. Microelectron Reliab 2007:47:1822-7.

[28] Pollak M. J Non Cryst Solids 1972;11:1-24.

[29] Arkhipov VI, Emelianova EV, Adriaenssens GJ. J Appl Phys 2003;93(10): 6150-3.

[30] Mott NF. Philos Mag 1969;19:835-52.

[31] Ambegaokar V, Halperin BI, Langer JS. Phys Rev B 1971;4(8):2612-20.

[32] Zaghloul U, Bhushan B, Pons P, Papaioannou GJ, Coccetti F, Plana R Nanotechnology 2011;22(3):035705.

[33] Rottenberg X, De Wolf I, Nauwelaers BKJC, De Raedt W, Tilmans HAC. J Microelectromech Syst 2007;16(5):1243-53.

[34] Bisquert J, Garcia-Belmonte G. J Appl Phys 2001;89:5657-62.

[35] Gelatos AV, Kanicki J. Appl Phys Lett 1990;57:1197-9. 